\title{
Structural Models based on Minimal Surfaces and Geodesics
}

Francisco González-Quintial. ETSA, Dpto. Arquitectura, Univ. del País Vasco. Email: francisco.gonzalez@ehu.eus

Andrés Martín-Pastor. (ETSIE, Dpto. Ingeniería Gráfica, Universidad de Sevilla)

\begin{abstract}
This article presents the results of research carried out with respect to the geometric, formal and structural adaptation of minimal surfaces. These surfaces were discretized into strips developable on geodesic curves, and then used for the construction of timber gridshells. For this project, both physical and virtual models derived from the same geometric models were used. The objective was to demonstrate the validity of the use of models and the transformation that modelling is undergoing due to the use of digital tools, both software and hardware. These include, on the one hand, drawing and analysis software and, on the other, digitally controlled fabrication tools. This research focuses specifically on the design and construction of the Scherk Pavilion, a space where the results of various experiments in which the common factor was the use of models was transferred to a real scale.
\end{abstract}

\section{Introduction}

In the era prior to architectural graphic representation as defined in the Renaissance, the function of models during design processes was ambiguous. It served as both as a test bed where a master builder, still "not an architect" in the Renaissance sense, simulated the physical behavior of their proposals. This included how they worked (Hauck et al. 2017) Due to the large scale of models, these were often visitable, and used as a kind of showroom (Garber 2009), where a potential patron could "tour" a project in a similar way 
to that in which a tour through an architectural space using immersive virtual reality is carried out today. The use of models was the instrument of global design prior to the use of floorplans (Fig. 1) and it defined the architectural element, as defined by Alberti, who formulated the bases of graphic architecture and of the "architect" as a designer rather than as a builder of architecture (Gentil 1998).

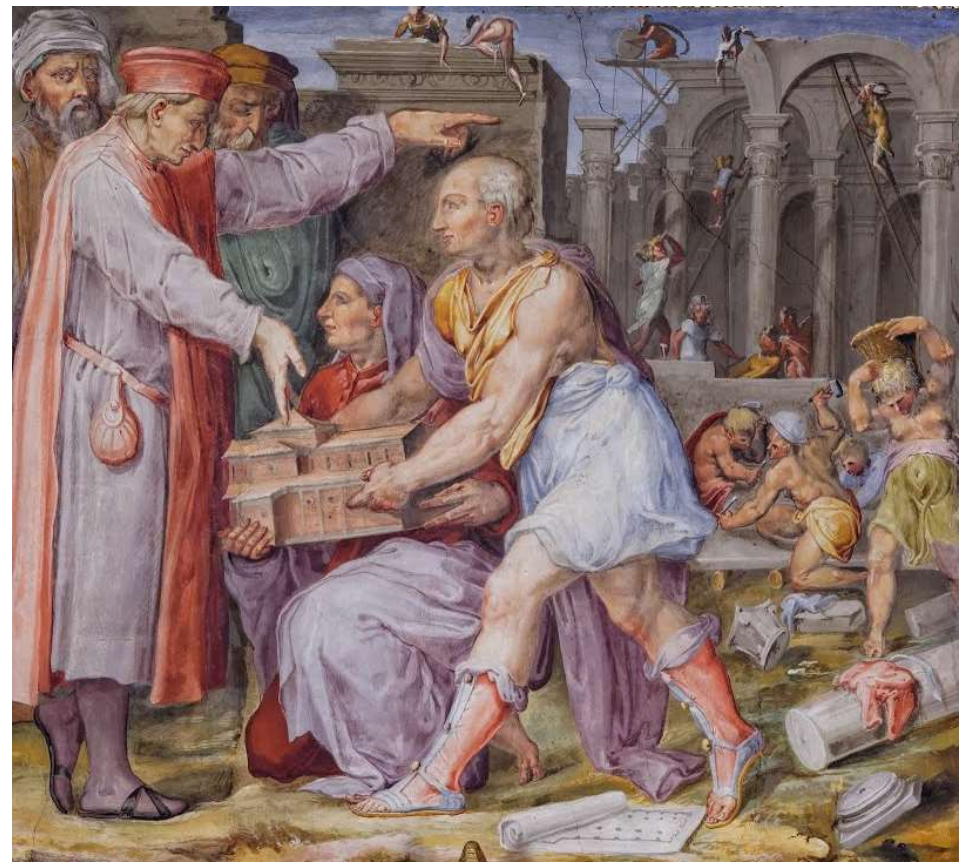

Fig. 1 Filippo Brunelleschi (kneeling) and Lorenzo Ghiberti show Cosimo de' Medici, counselled by Donatello and Michelozzo di Bartolomeo Michelozzi, a model of the Basilica di San Lorenzo, which is under construction in the background in 1429. Ceiling painting by Italian Mannerist painters Giorgio Vasari and Marco Marchetti da Faenza (1556-1558) in the Room of Cosimo the Elder in the Apartments of Pope Leo X in the Palazzo Vecchio in Florence. The painting was done after the event which it depicts and also after a shift from modeled to drawn architecture occurred. In the painting references to both types of architectural representation can be identified, although the model for representative purposes prevails

Of course, the field of application for models to represent the properties of real objects is limited. Leonardo proposed the study of the stress-strain relationships in bending, using models that he later collated graphically (Codex Matritensis, Tomo I, p. 137. National Library of Spain. Madrid. Bent Beam Models. http://leonardo.bne.es/index.html). As pointed out by Jacques Heyman, Galileo formulated the square-cube law that demonstrated the limitations of the use of models:

Other examples follow, all effectively demonstrating the square/cube law, culminating in Salviati's general statement about the impossibility of building enormous ships, palaces and temples - "nor could nature make trees of immeasurable size, because their branches would eventually fail of their own weight' (Heyman 1998: 9). 
Bill Addis added that certain elements are scalable while others are not:

The earliest mention of the non-linearity of scaling was by Galileo who noted (in what we now call the square:cube law) that while area increases with the square of the scale factor, the volume and hence the mass, increases as the cube of the scale. He used this observation to explain that the limited strength of bone material means the size of animals cannot be scaled up indefinitely (Addis 2013: 13).

Although we scale dimensions logically, structural function, in particular, exhibits a clear lack of linearity with changes of scale. A scale model can reproduce the behavior of a full sized object, to a greater or lesser extent, depending primarily on this object's structural behavior. That is, structural elements that depend on shape, such as arches and vaults, are almost perfectly scalable (Huerta 2004). Conversely, those elements that work mainly by section, such as beams, which, in other words, are subject mainly to bending stresses, do not offer the same degree of reliability when we analyze their behavior on different scales, although, as Hossdorf(1974) states, they do maintain a mechanical similarity.

Unlike traditional uses, models as we understand them today incorporate virtual space as a means of defining modelling itself from the most global point of view possible. We speak of virtual space and not of digital models, because we understand that digital codification existed prior to the appearance of computer instruments (Carpo 2011). Broadly speaking, we refer to virtual space closely connected to the different ways of representation, mainly in a graphic sense, of the models realised by using software tools, included any kind of numerical simulation, establishing a neat differentiation from the digital codification, despite the fact that it was digital as well. The true revolution caused by virtual digital space is the possibility of producing a large number of models, or derivations of models, in a very short period of time. In the words of Mario Carpo:

In this process of heuristic (not mathematical) optimization, every simulated model that was tried and discarded corresponded to a physical model that a traditional artisan would have made, tested, and likely broken in real life. Using digital simulations of structural performance, however, today we can make and break on the screen in a few hours more full-size trials than a traditional craftsman would have made and broken in a lifetime (Carpo 2017: 40). 
The extensive contemporary use of computers and digital virtual processes does not, however, completely overshadow another of the most powerful tools currently available for the management of models, that is, digital fabrication. Through computer-controlled digital tools we can return directly to physical models, completing design and manufacturing process through a return to pre-Renaissance, pre-Albertian architectural space, in which models reproduce a complex reality around architectural processes of design. This combination of digital software and hardware tools has thus meant both a synthesis and a true revolution in the classical concept of the model, allowing almost the same ease when handling both virtual and physical models and producing multiple approximations and variations of the same element.

In what follows, we present the different geometrical concepts, the development of the previous models, physical and numerical, and the progress of the final model of the pavilion that supported the research, which focused on the adaptation of the use of minimal surfaces discretized by using developable geodesic strips in order to construct timber gridshells.

\section{Geometric Concepts}

\section{Minimal Surfaces}

The first concept addressed in the research is that of minimal surfaces as structurally efficient forms. The concept, nuances and the use of minimal surfaces in architecture as “new forms" has many approaches (Emmer 1996). As a brief introduction, in Meusnier's formulation, minimal surfaces are those in which the mean curvature has vanished from a geometric point of view, which means that the curvature has the same value and opposite sign at each point. Physically, minimal surfaces are generated, in response to Plateau's problem (Pottman 2010), through a surface that covers, with the least possible extension, the space delimited by a rigid border that follows a closed curve without selfintersections. Surfaces produced through this approach do so as a sheet in which stresses are almost constant along the directions of curvature, and which, in the case of soap films, behave purely in traction. When we modify the boundary conditions, the edges are not sufficiently rigid, or we use a sheet with a degree of stiffness that is capable of absorbing stresses along the normal direction to the surface (i.e., a surface able to resist bending on its surface), purely tractional behavior disappears. Distortions appear as a result of bending in the surface itself and disturbances on free edges. 
These considerations have not excluded the use of this type of surface as a source of structural and architectural inspiration, as can be seen in the work of Frei Otto (Otto et al. 1998) and Sergio Musmeci (Marmo et al. 2019) among others. These architects made frequent use of models based on extensible surfaces as a principle for generating prototypes and the development of their ideas.

With the exception of the sphere, minimal surfaces are hyperbolic, anticlastic or negative curvature surfaces. While a sphere is a "minimal surface" in the sense that it minimizes the surface's area-to-volume ratio, it does not qualify as a minimal surface in the sense used by mathematicians. Hyperbolic shapes can be considered efficient from a structural point of view. We draw attention to the concrete shells by Félix Candela as an example of this type of structurally efficient form built in concrete (Faber 1963), particularly the hypars, - hyperbolic paraboloid sections - which, from a geometric point of view, have a great formal similarity with the minimal surfaces inside the edge of a warped quadrilateral.

The KnitCandela pavilion (Fig.2), is an evolution inspired by Candela's shells. It also uses minimal surfaces, though in this case as the basis of a textile formwork. It is on display as part of the first exhibition by Zaha Hadid Architects in Latin America, at the University Museum of Contemporary Art (MUAC) in Mexico City. In this example, it is interesting to observe the difference in the structural behavior of the fabric that makes up the permanent formwork of the surface, and that of the final concrete sheet.

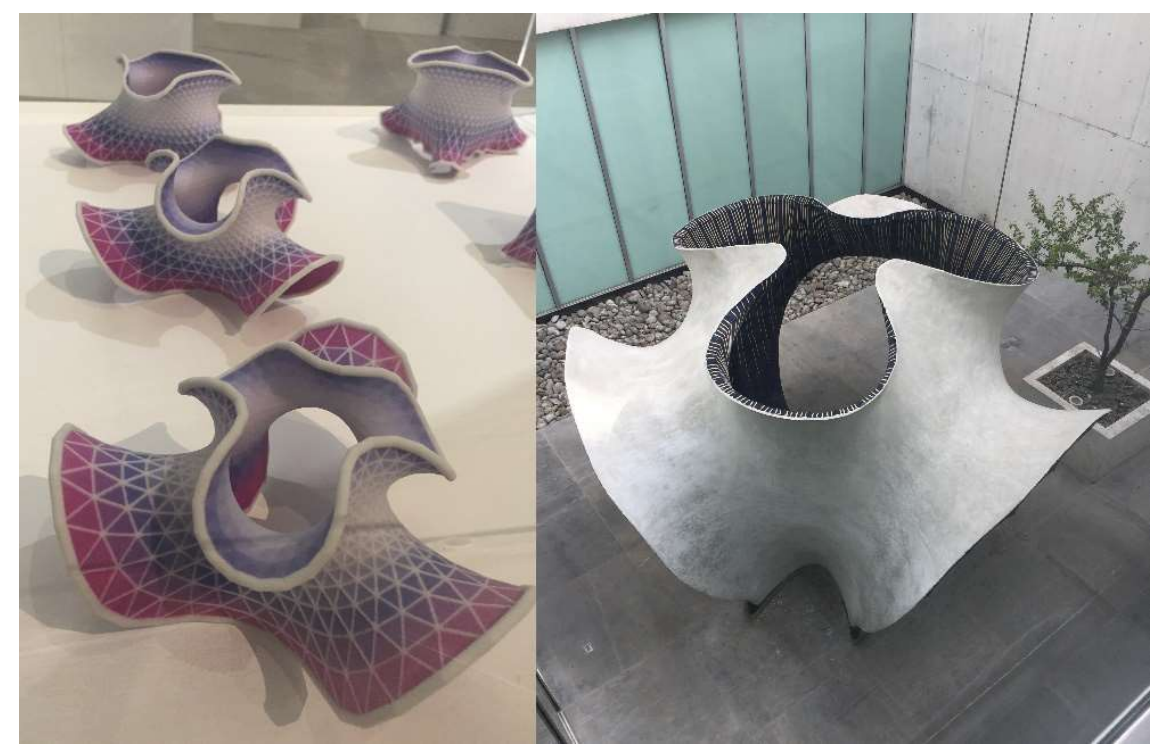

Fig. 2 KnitCandela pavilion. Philip Block Research Group and Zaha Hadid Architects at the MUAC in Mexico City. Images: authors 


\section{Developable strips on geodesic curves}

A second geometric concept that we used was the discretization of double curvature surfaces through the use of rectifying strips along geodesic curves on the surface. This turns out to be a very interesting procedure due to its high degree of efficiency with respect to the optimization of material.

A geometric approach is based on two interconnected ideas: on the one hand, the properties of a geodetic curve $\mathrm{G}$ on a surface $\mathrm{S} 1$; on the other hand, in the concept of the rectifying surface of a curve $\mathrm{C}$.

As is well understood, the geodesic $(G)$ is the "shortest path between two points (A) and (B) measured on the surface (S1)". On the other hand, the rectifying surface R of a curve $\mathrm{C}$ is "the developable surface that passes through a curve $(\mathrm{C})$ which has the property of transforming that curve into a straight line $\left(\mathrm{C}^{\prime}\right)$ once the surface $\left(\mathrm{R}^{\prime}\right)$ is deployed on a plane" (Izquierdo-Asensi 1985: 294).

Any curve drawn in this space allows a rectifying surface. We apply the concept of rectifying to the geodesic curve $\mathrm{G}$ drawn on a surface $\mathrm{S} 1$. In this way, the resultant curve is geodesic for both the surface S1 and the rectifying surface R, and likewise for the rectifying surface developed on the plane $\mathrm{R}^{\prime}$.

Thus, geodesic curves become the structure or guidelines for strips formed by rectifying surfaces. These R rectifying strips have two fundamental properties. They are strips circumscribed to the surface $\mathrm{S} 1$ on which the geodesic curve $\mathrm{G}$ is drawn, and they can be developed on the plane in the form of straight strips R'.

For manufacturing purposes, the straightness of the resultant strips facilitates construction based on rectangular elements that are straight and made of any material that can be bent in one direction. Keeping flat panels in mind as a common industrial construction material, use can be made of these with virtually no waste.

Straight rectifying strips can be bent without any stretching of the fibers and, therefore, without being subjected to any kind of twist. That is to say, theoretically, these strips suffer only minimal structural torsion. For this reason, they are suitable for use as structural elements with relativity thin cross sections, or their transverse dimension relative to their longitudinal dimension. 
This property is limited to a specific field of application. This implies that it is not always possible to find a suitable rectifying strip, from the point of view of fabrication, for every surface or even for any geodesic curve on a specific surface.

Furthermore, the width of strips is limited when we take into account a mixed deformation model including twist and bend. As a strip on a geodesic becomes wider, the elongation of fibers furthest from the axis becomes excessive. At this elongation limit, a strip tends to flex and not twist naturally, which could explain the appearance of the same geometric conditioning factors mentioned above in strictly rectifying strips. When a geodesic curve contains an inflection point, a change in curvature occurs. This results in a curve closer to the edge of regression of the rectifying surface that rests on it. This makes the developable surface constructively invalid, since it is not possible to extract a regular planar strip from its development. The use of a circumscribed developable surface on a geodesic is not possible on certain types of surface at certain points of their geodesic.

The models that we present here combine both geometric ideas, minimal surfaces and developable geodesic strips, in order to explore the stiffness and construction possibilities of reticular structures, also known as gridshells, made of wooden strips. This type of lightweight structure involves the discretization of surfaces through networks of curves along which several interconnected layers of lattices are supported. These generate lightweight, very stiff and aesthetically appealing constructions. The Manheim Multihalle by Frei Otto (Adriaenssens et al. 2014) is the pioneering example and served as inspiration for later examples, including the ribbed shells by Julius Natterer (Herzog et al. 2004) and the projects carried out by the IBOIS laboratory (Weinand 2017), as well as the Triaxial Gridshell made of eucalyptus constructed by PEMADE - Platform of Structural Timber Engineering (University of Santiago de Compostela) (Lara-Bocanegra et al. 2019), among other notable examples. Almost all of these, to a greater or lesser extent, use geodesic systems for surfaces and take advantage of the minimum torsion of curves to spatially arrange the lattices that compose them. Most of them also take elliptical or positive curvature surfaces as a starting point. However, no examples have been found of the use of minimal surfaces as the base geometry for this type of project.

\section{Preparatory Modelling}

As indicated, one of the concepts tested was the suitability of minimal surfaces for use as structural forms for the construction of timber gridshells. The starting point was, 
therefore, to obtain shapes that follow the morphology of surfaces of this nature, while also taking into account the deviation of constructed surfaces from purely mathematical models due to their physical behavior.

In this phase, an initial approach to the modelling of surfaces was carried out though classic soap bubble models, using soapy water and glycerin (Fig. 3). These generate minimal physical surfaces when rigid edges are imposed as surface boundaries. This process, despite being extremely simple and representing a basic concept, has been of great interest as a source of inspiration in work on tensile surfaces in general, through the study of minimal surfaces. Of course, physical reality is different from geometric reality. As Frei Otto points out, "surface structures in the form of geometrically exact minimal surfaces cannot be realized in practice except with soap membranes, or else in a more or less approximate form" (Otto et al. 1998: 246).

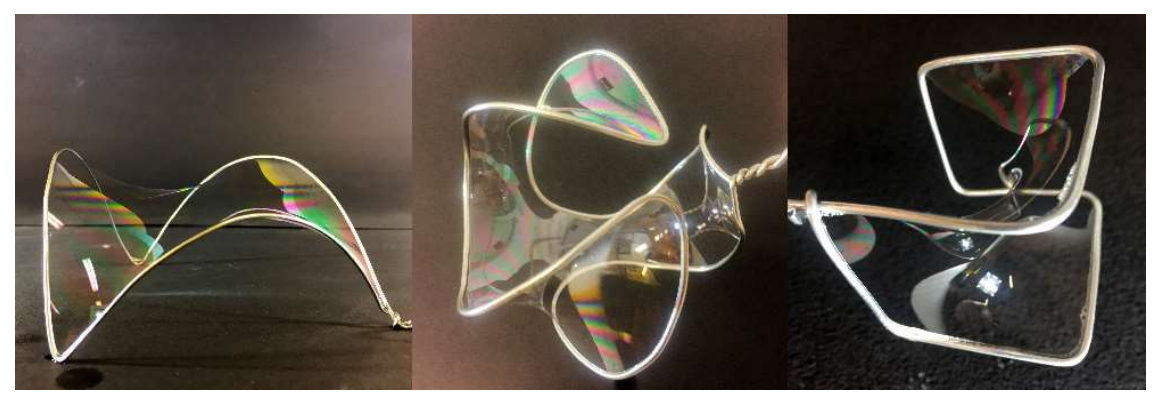

Fig. 3 Minimal surface physical models obtained using a bent wire without self-intersections, which acts as a rigid edge. Generation of the Scherk surface on the right. Images: authors

A physical model obtained using soap membranes is easily transferred into digital space. Using an algorithm generated in Kangaroo (https://www.food4rhino.com/app/kangaroophysics) a plug-in for Grasshopper (https://www.grasshopper3d.com/) (Fig.4), a representation of a tensile form is easily obtained by defining appropriate boundary conditions. In this phase, representations of a physical model can be much more accurate and precise, although at this point the design is in a purely mockup phase.

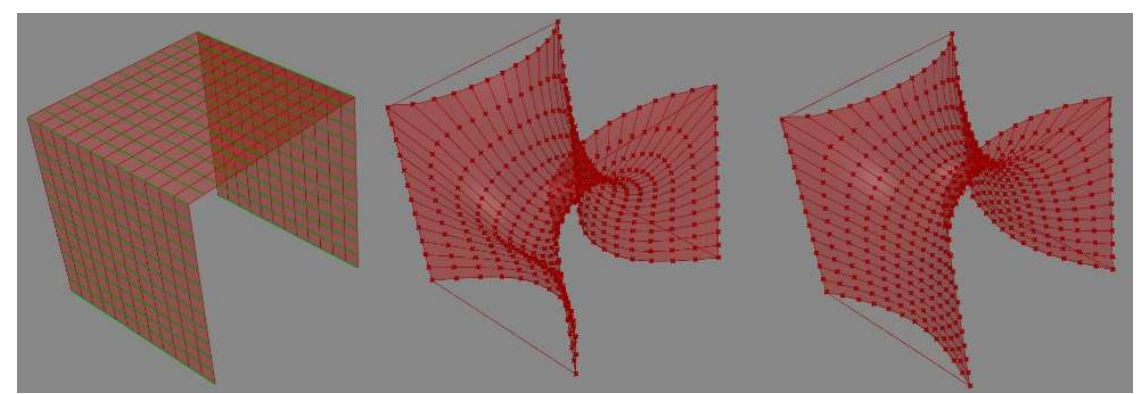

Fig. 4 By using Kangaroo, two families of curves are defined which act as cables with different stiffnesses, which facilitates the generation of a series of surfaces derived from the original geometric model based on the Scherk minimal surface. Images: authors 
From digital models, digitally fabricable files of the ideal shapes selected and modeled in Kangaroo were produced. These relatively small objects, produced using a 3D printer and replicated multiple times (Fig. 5), were used for both an intermediate study of a formal approach to design and for testing and explaining the concept of the geodetic curve. In this case, rubber bands were used to simulate the concept of geodesics. Due to their physical properties, elastic bands tend to adapt to a surface following the shortest possible path, that is, taking on the minimum possible tension. Due to this property, they serve to approximate the formal pattern of discretization of a surface by means of geodesic strips (Fig. 6).

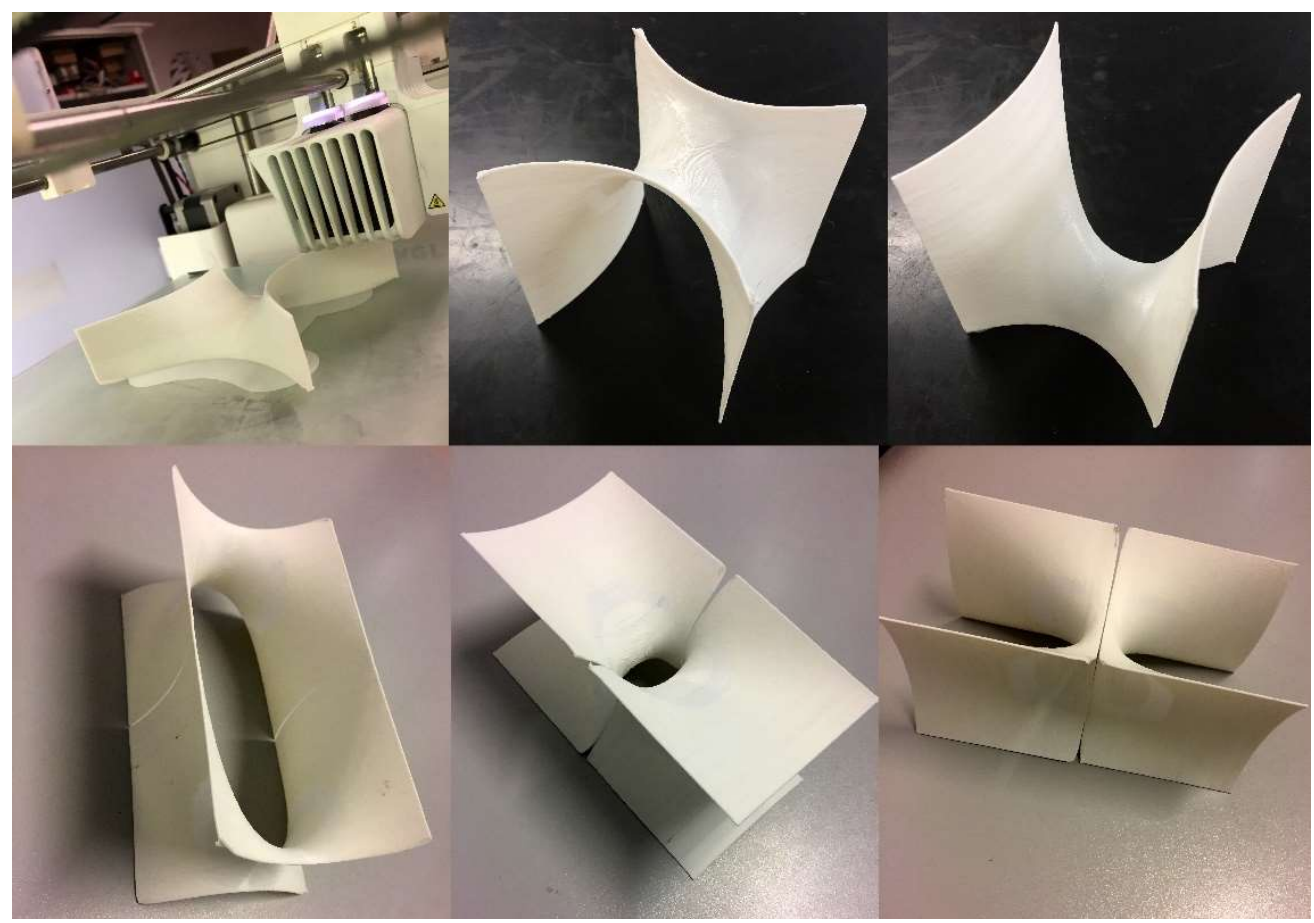

Fig. 5 3D printed physical models. The use of models as a design element and for compositional organization. Images: authors

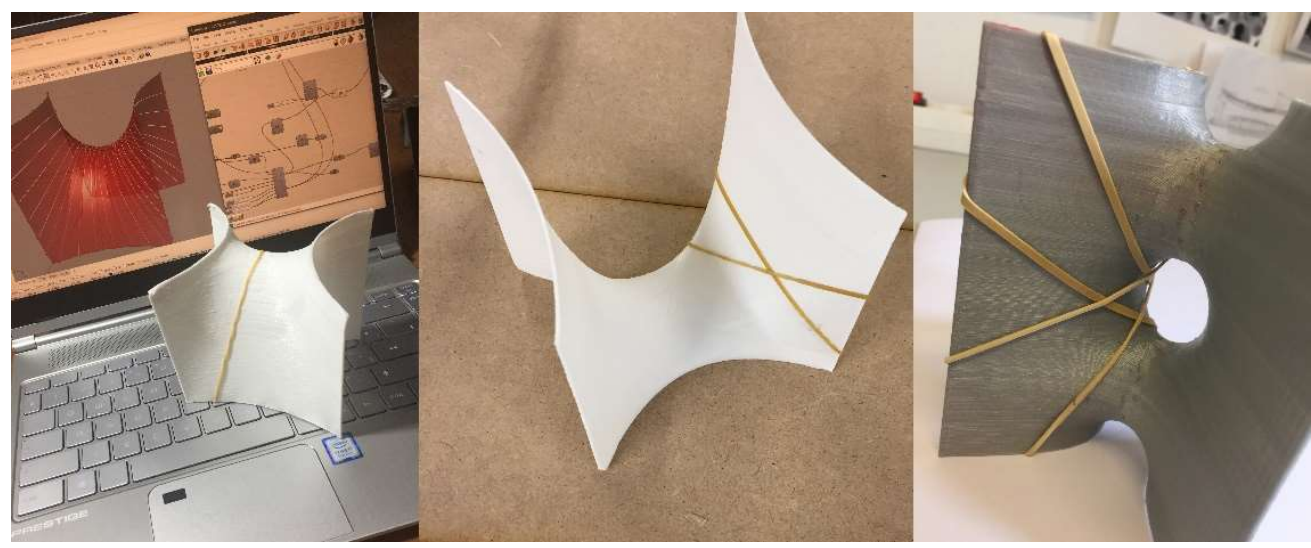

Fig. 6 Use of elastic bands on physical models as an aid to explain the behavior of geodesic curves and organization of a discrete mesh on a surface. Images: authors 
Different behavioral scale models were made to verify for constructional viability, stability, overall stiffness and the ability to geometrically reproduce a shape. Models have been spoken of as a system of preparation in architectural design, a way of understanding the geometry of the physical, even as a step prior to digital modelling. We propose a model that could be called a constructive approach. Models of an intermediate scale facilitate an anticipation of the reality of real materials and physical construction. Above all, however, they allow a faithful transcription from a conceptual drawn model to a physical model in terms of geometric issues. That is, a model is made at a scale where both the global shape and the particular constructive elements of the model are faithful to those of the full-scale architectural element. Similarly, the material used for models is based on the material intended for the full-scale project, scaling as far as possible for thickness and composition with a high degree of geometric rigor. The use of constructive approach scale models has been particularly useful in projects where construction problems can be anticipated and assembly difficulties anticipated, including the case of the Scherck Pavilion

Different cases have been approached following this procedure, using minimal surfaces as an initial sketch, and then discretizing them through the use of rectifying strips on geodesics (Fig. 7). All of these use one property of geodetic curves to locate the junction points between different strips. Specifically, a geodesic curve shares the normal with the surface of which it is geodesic. In other words, the vector normal to the curve, which is the radius of its osculating circle, coincides with the normal to the surface at each point on which we draw a geodesic. This allows a series of perforations to be placed at intersection points. At these holes, strips can be physically joined to each other. Beyond the manufacture of a single layer, this property of geodesic curves allows, as can be seen below, the production of multiple layers of strips, which significantly increases their rigidity. In this case the normal is still shared. Furthermore, the developable surface, in this case the surface circumscribed to the surface or rectifying strip, both of which are coincident and developable, maintain their developability when an offset to the surface is applied, that is, when the surface is translated in the direction normal to itself by a given value. 

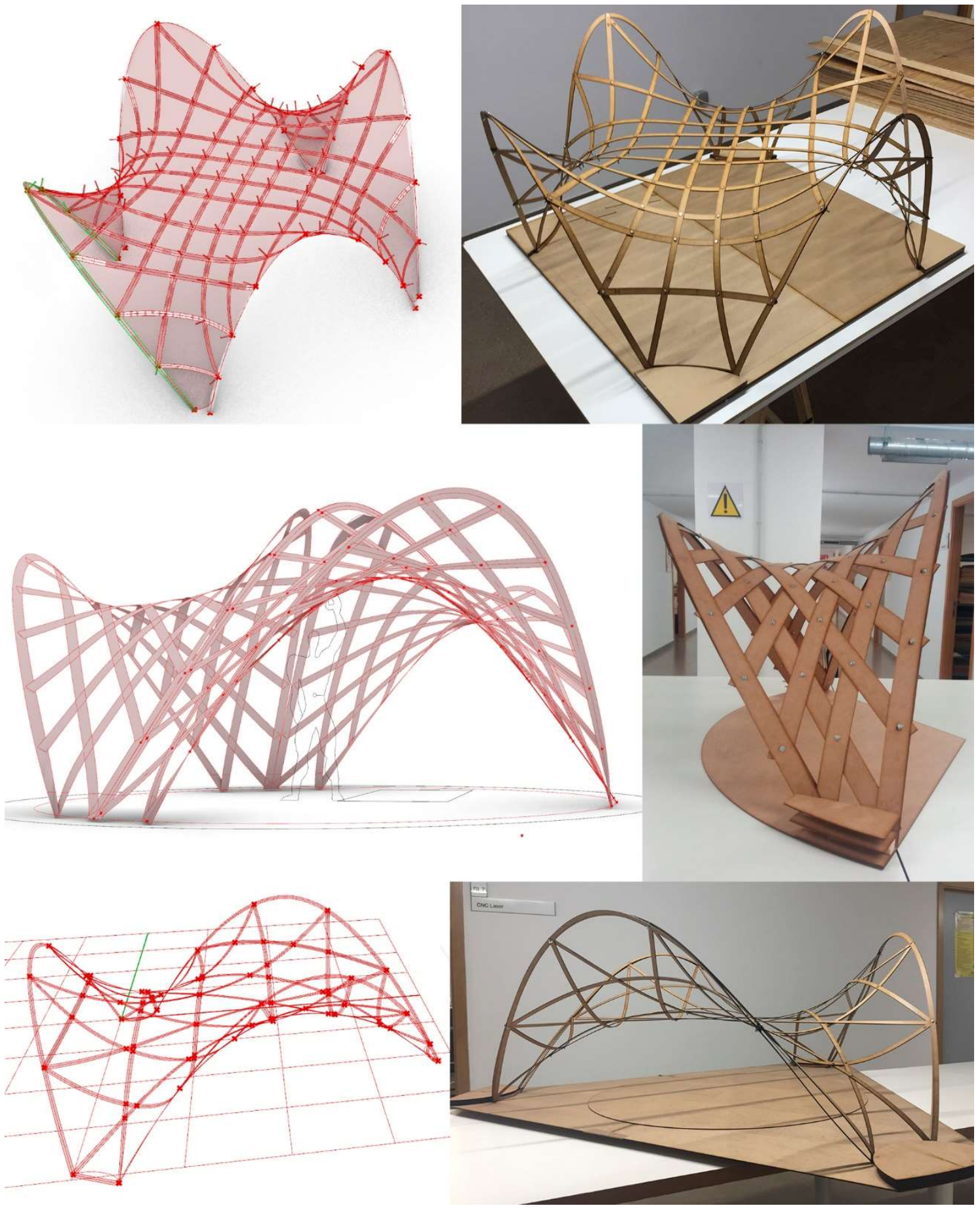

Fig. 7 Variations with different families of geodesic curves, double and triple directions. Images: authors

Beyond physical models, it seems reasonable and necessary to take advantage of geometric design models for the development of numerical models to facilitate a verification of tension and deformations in physical models. This step, while relatively easy and affordable for the analysis of simple geometric shapes, presents difficulties in the analysis of more complex geometries. There are different options for numerical 
calculation by finite elements. One of the most flexible in terms of complex geometries is Karamba (https://www.karamba3d.com/), an FEA modelling software that works in Grasshopper on the basis of a shared parametric model. This option, however, has limitations with respect to the modelling of the nature of wood-metal joints. These need to be understood as a model of springs or scissors where the transmission of forces in the joints follows a model more adapted to the physical interrelation of the two materials.

Another option is DLUBAL (https://www.dlubal.com/es), an FEA modelling software alternative. One of the models developed on the premises previously indicated was suitable for analysis with this software (Fig. 8). DLUBAL facilitates a modelling of the peculiarities of the behavior of the wood with a high degree of fidelity, especially with respect to the orthotropic behavior of the material. An important issue that can be modeled is the creep factor of material, which determines the dissipation of stress that appears in a piece as a product of active bending introduced during the bending of the elements that make up a structure. This factor limits short-term load bearing capacity. It also seemed essential to be able to produce a reliable numerical approach to the mechanical behavior of industrial wood-based materials, specifically that of multilayer plywood boards such as those used both in scale models and later in the construction of full-scale prototypes. Discontinuous, heterogeneous and anisotropic behavior can be modeled in the software used, so modelling at a numerical level does take into account the peculiarities of construction material.

Apart from purely mechanical issues, modelling presents an important problem of a geometric nature. There are several ways of transferring a shape from a design model to a numerical model, but none of these is capable of maintaining the geometric characteristics of the original surface. Geometric properties, developability in this case, can be lost in a transfer to a numerical model. Specifically, a surface defined by a strip which can be developed by rectifying is not kept as such in a numerical model. This implies an important gap in terms of accuracy when approaching the original model. The influence of this factor when translating results between physical and virtual models has not as yet been determined. 


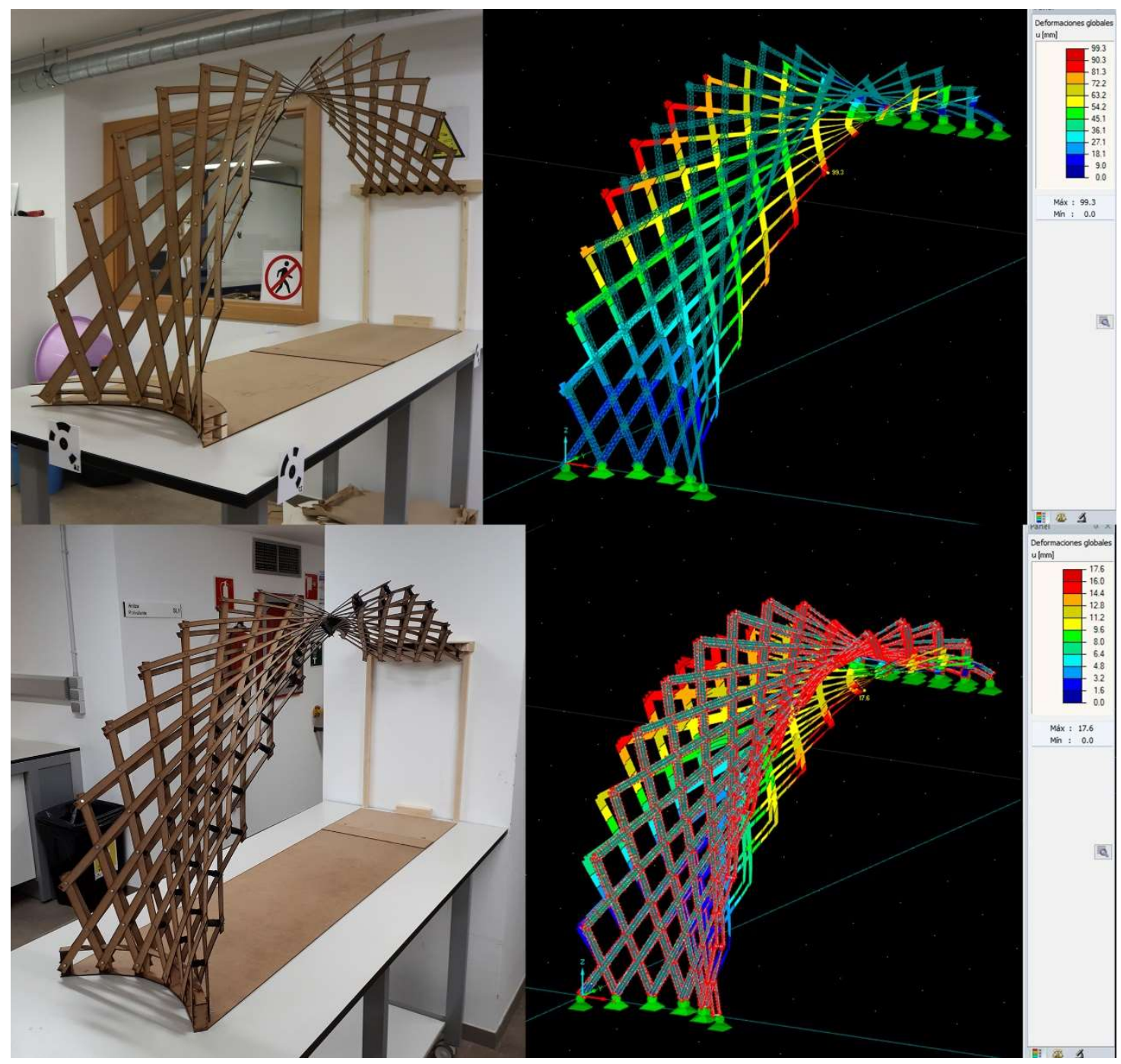

Fig. 8 A physical model and corresponding numerical analysis. In this example, single and double layer gridshell type surfaces were defined, and these geometries were then incorporated into FEA software, in this case DLUBAL. A significant increase in the stiffness of the structure is observed when a double layer is deployed, which reduces the maximum deformation by approximately $90 \%$. The main problem lies in the strictly geometric difference between the two models due to an inability to properly transcribe said geometry (Models made by Lorenzo Ghidini under the supervision of the authors).

\section{The Scherk Pavilion}

This project synthesized some of the abovementioned experiences and concepts, addressing both purely formal design issues and geometric aspects, as well as constructive solutions and assembly systems.

This project was carried out at FabLab Donostia, a digital fabrication laboratory at the Higher Technical School of Architecture of the University of the Basque Country, in the 
context of the I Lightweight Timber Structures Workshop organized by the Master in Design of Timber Structures (Fig. 9). The final object was the result of a four-day workshop in which a group of teachers and students worked on the development of an idea, fabrication of prototypes and final construction of the pavilion. The design aimed to verify the formal and structural possibilities of minimal discretized surfaces through the use of strips along geodesic curves on a surface.

The model was inspired by Scherk's first surface, discovered by Heinrich Ferdinand Scherk (1835; Dierkes et al. 2010). This is a minimal surface that can also be generated by translation, composed of two families of curves (catenaries of equal tension). Translated to the model, the two families of curves can be considered subject to different stresses. This varies their relative length and causes a controlled modification of the appearance of the surface itself in various models conditioned by the physical behavior of the curves, which is determined by variations in their rigidity.

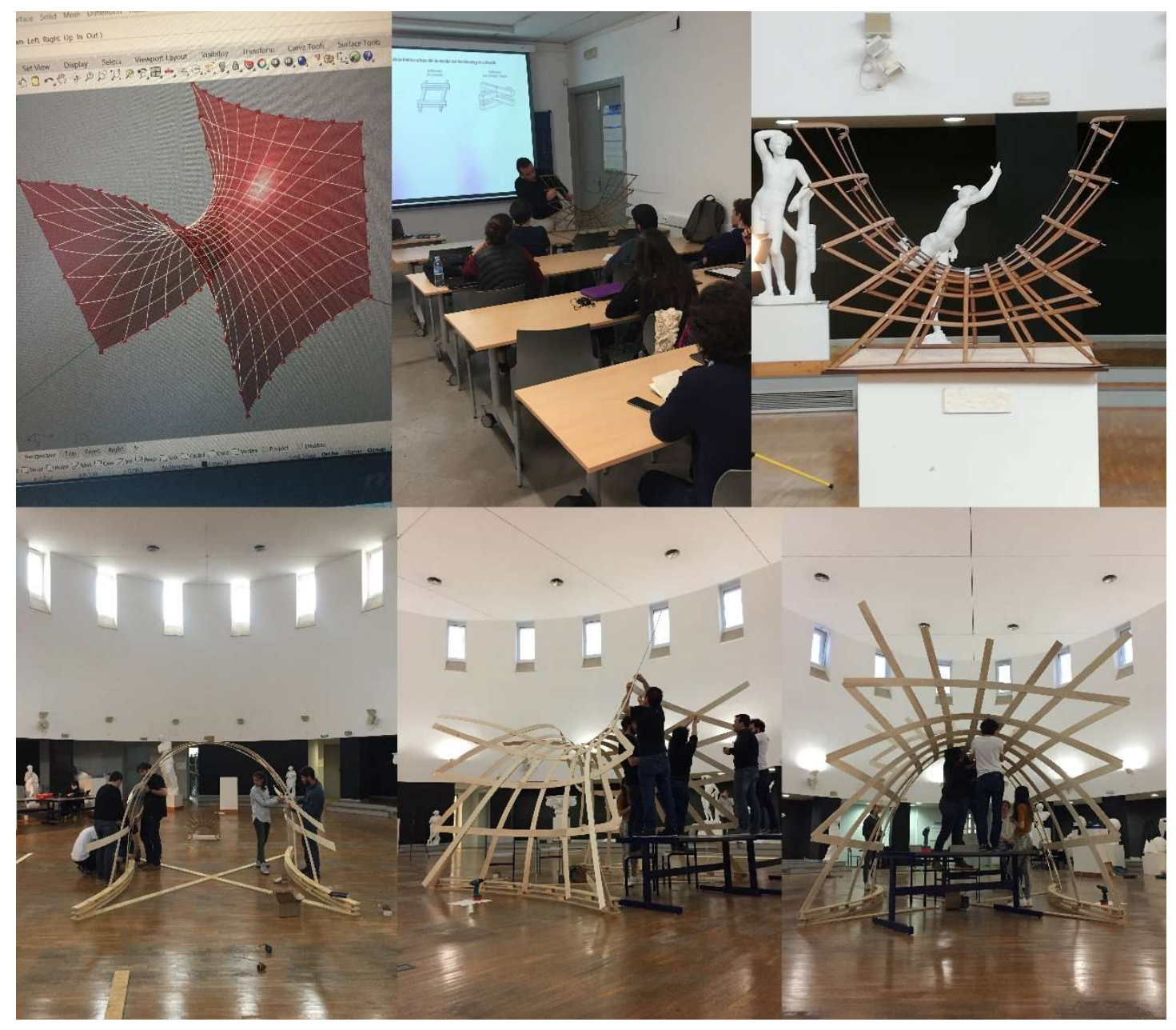

Fig. 9 Different phases of the constructive design approach to the pavilion and final construction. Authors 
The material used for the pavilion was $3 \mathrm{~mm}$ thick birch board, which facilitated the production of very flexible strips. Despite the fact that the curves involved had small radii of curvature, dry bending was used due to assembly being carried out indoors. Obviously, incorporating moisture into the material would lower its modulus of elasticity, allowing greater bending. This factor, combined with the proposal to push the mechanical capacities of the material to its limit, determined the choice of such thin material.

Initially, the design of the model called for a single layer, partly to keep the complexity of assembly under control and partly to avoid adding weight to the structure itself. Over the course of the workshop it was decided to incorporate a second layer, not duplicating the strips but shifting them on the surface and taking them to the middle of the opening of the initial grid. Obviously, this added significant weight, especially due to the resultant need to incorporate spacers made of a heavier material, as well as corresponding fastening elements. (Figs. 10, 11) Important questions about the joints and how the continuity of the material can be extrapolated depending on the scale is a part of the research in progress. We can anticipate that It depends more in the manufacturing of the material at industrial level than geometric questions.

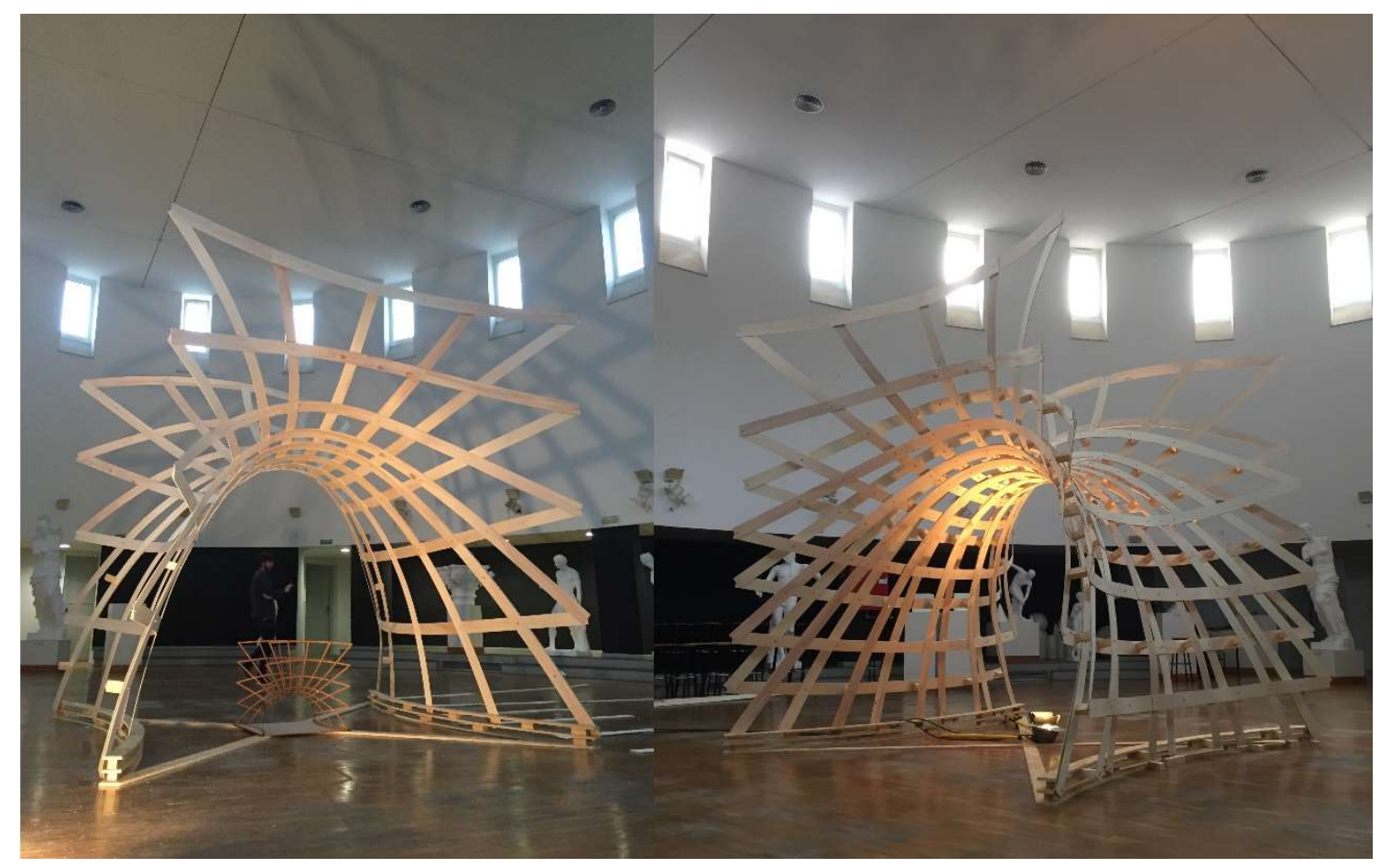

Fig.10 Final model. Single and double layered. Images: authors 


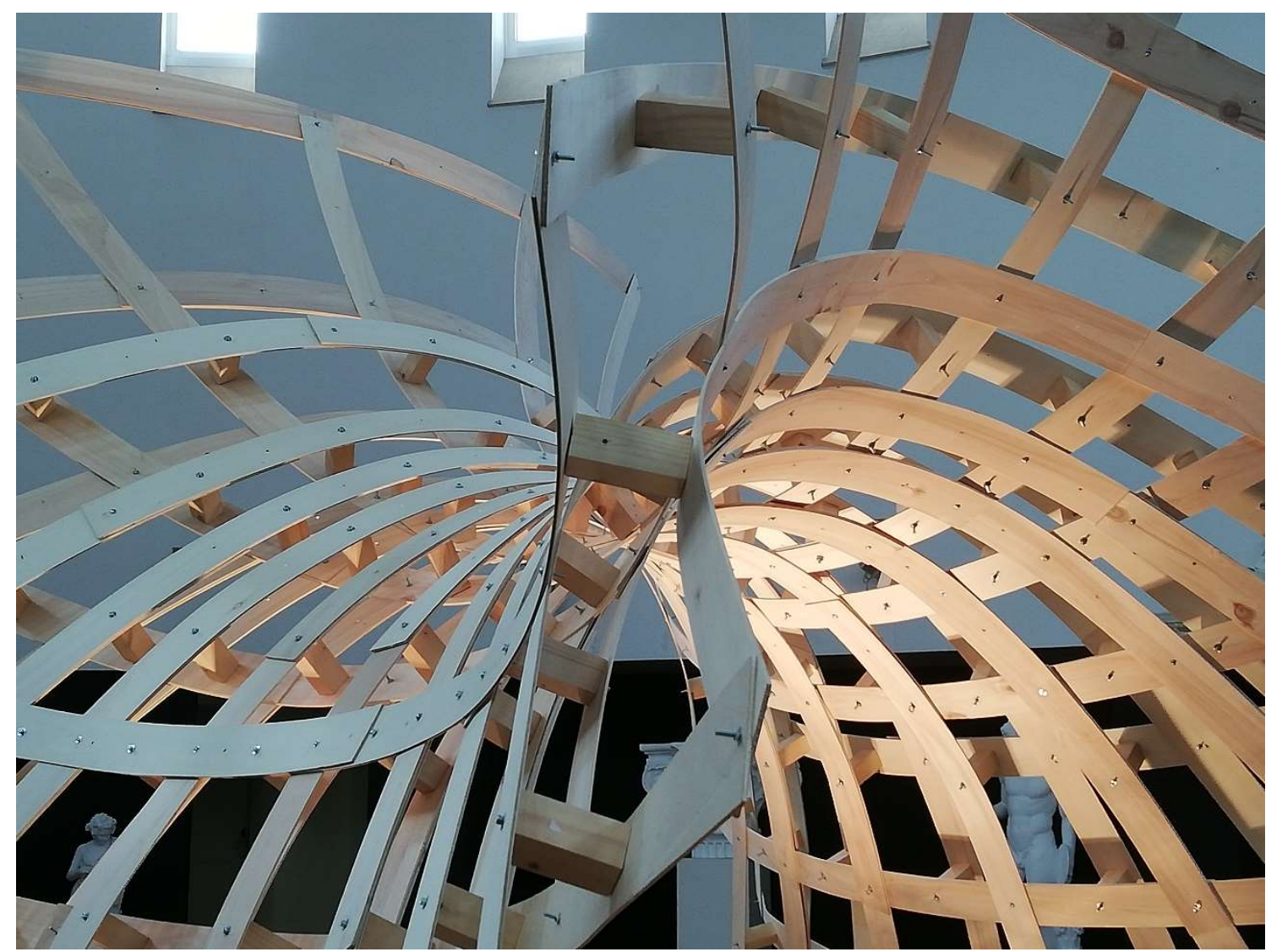

Fig. 11 Detail of the double-layered shell of the pavilion. The continuity of the strips is solved by overlapping, taking into account the continuity of the geometric curvature of them. Images: authors

The result, as mentioned above, was a more rigid shell model, subject to different deformations due to the increase in weight of the structure itself and the absence of a rigid edge that would have constrained the form and reduced the deformation of the strips.

It is important to recognize that the pavilion cannot be understood as a utilitarian construction, and from this point of view it is still just a model on a larger scale. It is a closer, more detailed approach to real construction through which to expand the workspace and experience the same ideas on a different scale. In this sense, the proliferation of this type of construction, mainly due to wider access to digital fabrication tools, must still be understood as an expansion of the scope of the use of models.

\section{Conclusion}

Models were one of the earliest means used to aid the design and development of architecture. Despite this, far from losing their relevance, their use has been enhanced 
through the incorporation of digital systems in processes of optimization, development and verification of architectural projects.

The results of the research process set out above illustrate the possibilities of structuring a system based on experimentation with the help of the physical and digital models. In a continuous process of return, there is a continual movement between scales and also between physical and virtual spaces in a process of both formal and structural optimization.

The model turns out to be today, as it was in another time, something much more complex and complete than the traditional architectural mockup. Models have maintained and even extended their relevance thanks to the emergence of different systems and tools, mainly digital, rather than to a change in the way models are used. The greatest revolution in this sense is of a quantitative nature. Together in a single digital file we have access to a repository of a large amount of information of multiple types. From graphic, geometric and representative elements, in addition to being a workbench where to test different aspects related to physical behavior, digital models facilitate the materialization of variations of a model in different formats and scales, thanks to digital manufacturing tools.

As to physical models, we can affirm that they turn out to be a key instrument in Researchby-Design processes in architecture (Lawson 2015) In both physical and virtual spaces, they are a direct way of doing research and acquiring knowledge, especially in preparatory and exploratory phases. Access to digital tools for the exploration of searching processes for shapes based on geometric foundations facilitates the elaboration of multiple models at different levels of precision and the verification of these primarily from the point of view of constructive suitability and geometric verification. This results in a high degree of accuracy.

Numerical models are a very powerful tools and we can recognize that they are capable of reproducing intrinsic properties of materials in a much more efficient way. This overcomes problems of physical models, specifically in terms of a lack of scalability for certain structural behaviors. However, we have verified that these models are not able to correctly reproduce geometries with a high degree of complexity, as is the case at hand. Therefore, it is necessary to validate numerical models by checking the data extracted from these through physical models. 
A next step would be the verification of numerical results and a comparison of these results with those derived from physical models at different scales. The objective would be to characterize a pattern of mechanical behavior validating the results of numerical models. One aspect that needs to be considered for future development is the synchronization of physical and numerical models. The first step has already been carried out, in the verification of the validity of numerical models in relation to physical models by photogrammetric surveys of physical models. These have allowed a superposition of results offered by numerical and physical models separately. This, together with a characterization of different construction materials with greater precision - an issue outside our area of work - could lead to adjusting both approaches so that a single model of global validity is achieved.

\section{References}

Addis, Bill. 2013. "Toys that save millions". A history of using physical models in structural design. Structural Engineer 91: 12-27.

Adriaenssens, Sigrid, Philippe Block, Diederik Veenendaal, Chris Williams. 2014. Shell Structures for Architecture: Form Finding and Optimization. London: Routledge.

Carpo, M. 2011. The alphabet and the Algorithm. Cambridge, Mass.: MIT Press.

Carpo, Mario. 2017. The Second Digital Turn: Design Beyond Intelligence. Cambridge, Massachusetts: The MIT Press.

Dierkes, Ulrich, Stefan Hildebrandt, Friedrich Sauvigny. 2010. Minimal surfaces. Rev. and enl. 2nd ed. Heidelberg: Springer.

Emmer, Michele. Architecture and Mathematics: Soap Bubbles and Soap Films. In, Nexus: Architecture and Mathematics, ed. Kim Williams, pp. 53-65. Fucecchio (Florence): Edizioni dell'Erba.

Faber, Colin. 1963. Candela, the shell builder. New York: Reinhold.

Garber, Richard. 2009. Alberti's Paradigm. Architectural Design 79(2): 88-93.

Gentil Baldrich, José Maria. 1998. Traza y modelo en el Renacimiento. Sevilla: Instituto Universitario de Ciencias de la Construción.

Hauck, Anthony. Michael Bergin, Phil Bernstein. 2017. The Triumph of the Turnip. In: Fabricate 2017, eds. Achim Menges, Bob Sheil, Ruairi Glynn and Marilena Skavara, 16-21. London: UCL Press.

Herzog, Thomas, Julius Natterer, Roland Schweitzer, Michael Volz, Wolfgang Winter. 2004. Timber Construction Manual. Basel, Munich: Birkhäuser, Edition Detail.

Heyman, Jacques. 1998. Structural analysis: a historical approach. Cambridge: Cambridge University Press.

Hossdorf, Heinz. 1974. Model Analysis of Structures. New York: Van Nostrand Reinhold.

Huerta, Santiago. 2004. Arcos, bóvedas y cúpulas: geometría y equilibrio en el cálculo tradicional de estructuras de fábrica. Madrid: Instituto Juan de Herrera. 
Izquierdo Asensi, F. 1996. Geometría descriptiva superior y aplicada. 4th rev. ed. Paraninfo.

Lara-Bocanegra, Antonio José, Antonio Roig Vena, Almudena Majano-Majano, Manuel Guaita. 2019. From eucalyptus to elastic gridshells. IASS Annual Symposium 2019 Structural Membranes 2019 Form and Force. Barcelona (Spain), 7-10/10/2019.

Marmo, Francesco, Cristoforo Demartino, Gabriele Candela, et al. 2019. On the form of the Musmeci's bridge over the Basento river. Engineering Structures 191: 658-673.

Lawson, Bryan R. 2015. Book Review of Design Research in Architecture: An Overview by Frasier Murray. Design Studies 36: 125-130.

Otto, Frei, Klaus Bach, Frieder Klenk, et al. 1998. Prinzip Leichtbau = Lightweight principle. Form-Kraft-Masse 4 / Form-Force-Mass 4. Mitteilungen des Instituts für leichte Flächentragwerke (IL 24). Stuttgart: Krämer, .

Scherk, H. F. 1835. Bemerkungen über die kleinste Fläche innerhalb gegebener Grenzen. Journal für die reine und angewandte Mathematik 13: 185-208.

Weinand, Yves. ed. 2017. Advanced Timber Structures: Architectural Designs and Digital Dimensioning. Basel: Birkhäuser. 